\title{
Characteristics of the electric field accompanying a longitudinal acoustic wave in a metal. Anomaly in the superconducting phase
}

\author{
Yu. A. Avramenko, ${ }^{1}$ E. V. Bezuglyi, ${ }^{1}$ N. G. Burma, ${ }^{1}$ I. G. Kolobov,${ }^{1}$ V. D. Fil ${ }^{1}{ }^{1}$ O. A. Shevchenko, ${ }^{1}$ and V. M. Gokhfeld ${ }^{2}$ \\ ${ }^{1} B$. Verkin Institute for Low Temperature Physics and Engineering, \\ National Academy of Sciences of Ukraine, pr. Lenina 47, 61103 Kharkov, Ukraine \\ ${ }^{2}$ A. A. Galkin Donetsk Physicotechnical Institute, ul. R. Lyuksemburg 72, 83114 Donetsk, Ukraine
}

\begin{abstract}
The temperature dependence of the amplitude and phase of the electric potential arising at a plane boundary of a conductor when a longitudinal acoustic wave is incident normally on it is investigated theoretically and experimentally. The surface potential is formed by two contributions, one of which is spatially periodic inside the sample, with the period of the acoustic field; the second is aperiodic and arises as a result of an additional nonuniformity of the electron distribution in a surface layer of the metal. In the nonlocal region the second contribution is dominant. The phases of these contributions are shifted by approximately $\pi / 2$. For metals in the normal state the experiment is in qualitative agreement with the theory. The superconducting transition is accompanied by catastrophically rapid vanishing of the electric potential, in sharp contrast to the theoretical estimates, which predict behavior similar to the BCS dependence of the attenuation coefficient for a longitudinal sound.
\end{abstract}

\section{INTRODUCTION}

When an elastic wave propagates in a metal, a perturbation of its electron subsystem occurs which compensates the tendencies of the ionic displacements to disrupt the charge neutrality or the balance between the ion and electron currents. At high temperatures or in dirty samples (the so-called local limit, determined by the condition $q l \ll 1$, where $q$ is the acoustic wave vector and $l$ is the electron mean free path) the electron collision frequency is high enough to maintain local equilibrium at any point of the deformed lattice. In the limit $q l \ll s / v_{F}$ ( $s$ and $v_{F}$ are the sound velocity and Fermi velocity) the electric field arising in the metal has a purely inertial nature - the acoustic analog of the Stewart-Tolman effect.

In the opposite limiting case ( $q l \gg 1$, nonlocal limit) the collisions can no longer maintain local equilibrium, and additional electric fields are produced in the metal, bringing about the required adjustment of the electrons to the moving lattice. In zero magnetic field the polarization of the electric field of an elastic wave propagating in an isotropic metal or along a high-symmetry direction is parallel to the ionic displacements. When a magnetic field is turned on, the Lorentz force exerted on the electrons moving with the lattice gives rise to a Hall component of the alternating electromagnetic field, the existence of which is no longer dependent on strict requirements on the purity or temperature of the sample.

The opposite process also occurs: the generation of acoustic vibrations when a polarized electromagnetic field is excited in the metal in a suitable way. On the whole, these phenomena - the acoustoelectric and electroacoustic transformations are well known and widely used both in scientific experiments and for contactless excitation of sound in technical applications (see, e.g., the review ${ }^{\underline{1}}$ ).

However, as far as the authors are aware, the experimental studies have always been done for electromagnetic fields in which the electric field vector was orthogonal to the wave vector of the elastic wave. ${ }^{2}$ The latter is apparently due to the fundamental differences in the behavior of the fields of transverse and longitudinal polarization at the interface between the metal and free space (or an insulating medium). For normal incidence of a transverse elastic mode on the interface the electromagnetic field in the metal is naturally "matched" with the electromagnetic field in the vacuum (insulator). In other words, in this geometry an electromagnetic wave is radiated from the metal; this wave can easily be detected by a suitably oriented antenna of the flat-coil type. This same antenna can also be used to generate a transverse elastic wave in the metal.

An electromagnetic wave with electric field polarized along the wave vector does not exist. Therefore, in the case of normal incidence of a longitudinal acoustic wave on the interface, electromagnetic radiation from the sample is absent in principle. Nevertheless, it is possible to detect the electric field $\mathbf{E}$ due to a longitudinal acoustic wave. Since $\operatorname{div} \mathbf{E} \neq 0$ in a longitudinal wave, uncompensated charge and the accompanying potential appear both in the bulk of the metal and on

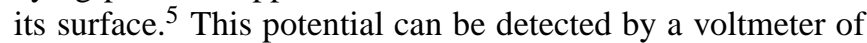
suitable sensitivity. One of the authors (V.M.G.) long ago had analyzed a method of linear electroacoustic conversion with the use of a contactless capacitive driver. - Here, when an alternating voltage is applied to the exciting plate of the capacitor, an electric charge is induced beneath it on the surface of the metal, and the nonlocal interaction of this charge with the lattice excites a longitudinal elastic wave of the same frequency. A detailed experimental check of Ref. 6 has not been made, possibly because of the rather stringent requirements on the purity of the metal. The limiting case of a capacitive driver (receiver) is a galvanic contact, which is what was mainly used in the present study. Finally, the presence of the contact on the interface alters the boundary conditions for the elastic deformations; however, it turns out that the latter leads to a slight increase in the conversion efficiency and in general relaxes the requirements on the purity of the material under study. For this reason it is possible to carry out an experiment on practically all reasonably pure metals provided that some relatively simple requirements are met as to the nonlocality of the interaction of the electrons with the elastic field $(q l>1)$.

The structure of this paper is as follows. In Sec. 2 a theory of the acoustoelectric conversion is given for the simplest case - a conducting half space (one-dimensional problem) in 
the approximation of an isotropic dispersion relation for the carriers and their "specular" reflection by the interface. The electric potential accompanying an elastic wave in the metal is a sum of two contributions. Besides the well-known forced solution, which repeats the profile of the elastic deformations, in the nonlocal limit there is also a substantial quasiperiodic term due to the perturbation of the ballistic motion of the electrons by the surface of the sample.

In Sec. 3 we present the results of experimental studies of the temperature dependence of the aforementioned electric potential in metals of different degrees of purity $(\mathrm{Ga}, \mathrm{W}$, $\mathrm{Al}$ ) in the normal state. On the whole, the temperature dependences of the amplitude and phase of the potential show an acceptable qualitative agreement with the theoretical estimates; this can be regarded as experimental confirmation of the theoretical ideas proposed here.

In Sec. 4 we discuss the evolution of the measured electric potential at the superconducting transition. Contrary to the expectation of a rather slow decrease in the recorded value below $T_{C}$ (like the behavior of the longitudinal sound attenuation coefficient), a much sharper decrease in the amplitude of the potential is observed.

\section{THEORY OF ACOUSTOELECTRIC CONVERSION IN THE CASE OF NORMAL INCIDENCE OF A SOUND WAVE ON AN INTERFACE}

Let us consider a metallic half space $x \geq 0$. Suppose that a longitudinal elastic wave $u_{x}=u_{0} \exp (-i \omega t-i q x)$ comes in from the interior of the sample and is reflected from the sample boundary with a reflection coefficient $R$ (we neglect the sound attenuation). Near the boundary the displacement field and the deformation field are formed as a result of the interference of the incident and reflected waves, independently of the regime (pulsed or continuous). The resultant field is expressed in terms of the amplitudes of the displacement $u(0)$ and deformation $u^{\prime}(0)$ at the interface. In particular, for the deformation field that we will be interested in below we have

$$
\frac{d u_{x}(x)}{d x} \equiv u_{x x}(x)=u^{\prime}(0) \cos q x-q u(0) \sin q x .
$$

The relation between $u(0)$ and $u^{\prime}(0)$ is determined by the boundary conditions

$$
\frac{q u(0)}{i u^{\prime}(0)}=\frac{1+R}{1-R} \equiv C .
$$

For a contact with an elastically uniform, nonattenuating medium the parameter $C$ is equal to the ratio of the acoustic impedances of the metal and of the medium in contact with it (in particular, $C=\infty$ for a contact with the vacuum). In the case of an attenuating medium the parameter $C$ can be complex-valued.

The deformation-induced deviation $\psi\left(x, v_{x}\right) \partial f_{0} / \partial \varepsilon$ of the electron distribution function from its equilibrium value $f_{0}$ is described by the kinetic equation ${ }^{7}$ (we neglect the inertial field):

$$
v_{x} \frac{\partial}{\partial x}(\psi-e \varphi)-i \widetilde{\omega} \psi=i \omega \Lambda_{x x} u_{x x}
$$

Here $\widetilde{\omega} \equiv \omega+i / \tau$ ( $\omega$ is the angular frequency, and $\tau$ is the electronic relaxation time), $\Lambda_{x x}$ is the corresponding component of the deformation potential tensor $\Lambda_{i k}=\lambda_{i k}-\left\langle\lambda_{i k}\right\rangle /\langle 1\rangle$, $\lambda_{i k}$ is the "bare" deformation potential, $\varphi(x)$ is the electric potential in the field of the elastic wave, $v_{x}$ is the $x$ component of the Fermi velocity $v_{F}$ of the electron, and the angle brackets denote averaging over the Fermi surface with a weight $v_{F}^{-1}$ :

$$
\langle A\rangle \equiv \frac{2}{h^{3}} \int \frac{A d S}{v_{F}} .
$$

It is important to note that the quantity $e \varphi(x)$ appearing in Eq. (3) is actually the total electrochemical potential of the electrons, including, in addition to the "true" electric potential, the change of the chemical potential $u_{x x}\left\langle\lambda_{x x}\right\rangle /\langle 1\rangle$ due to the change of the electron spectrum in the elastic deformation field $\underline{\underline{7}}$ The difference of the electrochemical potentials at different points of the sample is a source of real emf that can be registered by a voltmeter, and it, of course, vanishes at equilibrium, e.g., in the limit of a static deformation $(\omega \rightarrow 0)$. At the same time, the gradient of the "true" electric potential, which compensates the deformation contribution to the electrochemical potential and brings about an adjustment of the electron density to the spatial variations of the ion density is always nonzero in a nonuniformly deformed sample (physically this effect is analogous to a contact potential difference). The presence of this potential in a nonuniformly deformed metal (including in the elastic field of a sound wave) gives rise to uncompensated charges with a density $\delta n=r_{D}^{2}\left\langle\lambda_{x x}\right\rangle \nabla^{2} u_{x x} \sim\left(q r_{D}\right)^{2} q u n$, where $n$ is the total electron density, $q$ is the characteristic wave number of the deformation, and $r_{D}$ is the screening radius of the longitudinal field $\left(r_{D}^{-2}=4 \pi e^{2}\langle 1\rangle\right)$, which in "good" metals is of the order of the lattice constant. Such a "charge density wave" accompanying the propagation of a sound wave in the general case contains a nonequilibrium contribution due to the disruption of the spatial uniformity of the electrochemical potential $e \varphi(x)$; this contribution is proportional to the frequency of the sound (see below) but in the majority of cases it is small $\left(\sim s / v_{F}\right)$ compared to the "adiabatic" component mentioned above. To avoid misunderstandings we should say that, because of the small value of the uncompensated charge $(\delta n \ll n)$ the potential $\varphi(x)$ can, of course, be calculated from the condition of electrical neutrality $\langle\psi\rangle=0$, where $\langle\psi\rangle$ has the meaning of a nonequilibrium admixture to the charge density which is "adiabatically" modulated by the elastic field.

Far from the boundary $(x \gg l)$ one can assume that the functions $\psi(x)$ and $\varphi(x)$ are periodic, with the same spatial period as $u(x), \frac{7}{,}$ and in this case Eq. (3) reduces to an algebraic equation. Near the interface (at distances $x \leq l$ from it) the electron distribution differs substantially from periodic, and the problem of finding $\psi(x)$ and $\varphi(x)$ is complicated. It can be solved relatively simply by the Fourier method if the so-called specular boundary condition is imposed on the function $\psi(x)$ :

$$
\psi\left(+0, v_{x}\right)=\psi\left(+0,-v_{x}\right) .
$$

We emphasize that the condition of "specular" reflection from the moving boundary can be written in the form (4) only 
in a comoving reference frame, in which there is no current through the boundary $\left(\left\langle v_{x} \psi\left(0, v_{x}\right)\right\rangle=0\right)$, and the scattering of the electrons is elastic. Since the potential measurement is actually done at a moving boundary, all of the calculations below pertain to the comoving system, in which the form (3) of the linearized kinetic equation is preserved.

Let us continue the functions $u_{x x}(x)$ and $\varphi(x)$ evenly onto the semiaxis $x<0$ (this will be denoted below by a superscript $S$ ). Then from the form of Eq. (3) (in view of the evenness of $\Lambda_{x x}\left(v_{x}\right)$ it is symmetric with respect to a simultaneous change of the signs of $x$ and $v_{x}$ ) and condition (4) it follows that the unknown function $\psi(x)$ should be continued to $x<0$ without a discontinuity. As a result, its transform is equal to

$$
\psi_{k}=\frac{k v_{x}\left(\varphi^{S}\right)_{k}-\omega \Lambda_{x x}\left(u_{x x}^{S}\right)_{k}}{k v_{x}-\widetilde{\omega}} .
$$

The Fourier transform of the electric potential $\left(\varphi^{S}\right)_{k}$ of interest is found from the condition of electrical neutrality of the metal, $\left\langle\psi_{k}\right\rangle=0$ :

$$
\left(\varphi^{S}\right)_{k}=\left(u_{x x}^{S}\right)_{k}\left\langle\frac{\omega \Lambda_{x x}}{k v_{x}-\widetilde{\omega}}\right\rangle\left\langle\frac{k v_{x}}{k v_{x}-\widetilde{\omega}}\right\rangle^{-1} \equiv\left(u_{x x}^{S}\right)_{k} R_{k}
$$

where $\left(u_{x x}^{S}\right)_{k}$ is the transform of the deformations (1),

$$
\left(u_{x x}^{S}\right)_{k}=q\left[\frac{q u(0)-i u^{\prime}(0)}{k^{2}-(q+i 0)^{2}}+\frac{q u(0)+i u^{\prime}(0)}{k^{2}-(q-i 0)^{2}}\right] .
$$

Integration over the Fermi surface in (6) leads to branch points $k= \pm K\left(K=\tilde{\omega} / v_{F}\right)$ of the kinetic coefficient $R_{k}$; these singularities are in addition to the "acoustic" poles $k=$ $\pm(q \pm i 0)$ of expression (7). Inversing the Fourier transform, we find that in addition to the periodic forced solution, which is determined by the poles (the $q$ contribution), the function $\varphi(x)$ also contains an aperiodic term, whose amplitude is a complicated function of $x$ and whose phase $(\sim K x)$ is determined by the Fermi velocity ( $K$ contribution).

The asymptotics of the solutions of this type at large depths have been discussed previously on more than one occasion in the analysis of the propagation of electron quasiwaves in a metal ${ }^{6,8,9}$ In the present paper we will be interested in the relationship of the $K$ and $q$ contributions to the electric potential $\varphi(0)$ measured at the boundary of the metal and their respective temperature dependences.

Let us see what the solution (6) gives in the simple case of a quadratic isotropic dispersion relation of the charge carriers, $\varepsilon=p^{2} / 2 m$, when the deformation potential can be represented in the form ${ }^{7}$

$$
\Lambda_{x x}=L\left(3 v_{x}^{2} / v_{F}^{2}-1\right) .
$$

In this case the kinetic factor in (6) is equal to

$$
\begin{aligned}
& R_{k}=\frac{\omega L}{\widetilde{\omega}} \Phi(z), \quad \Phi(z)=\frac{3}{z^{2}}-\frac{\ln \frac{1-z}{1+z}}{2 z+\ln \frac{1-z}{1+z}}, \\
& z=\frac{k v_{F}}{\widetilde{\omega}}=\frac{k l}{\omega \tau+i} .
\end{aligned}
$$

To find the coefficient of proportionality between $u_{x x}(x)$ [see Eq. (1)] and the $q$ contribution to $e \varphi(x)$, it is sufficient to substitute $k=q$ in (9); thus, in the $x$ representation we obtain

$$
e \varphi_{q}(x)=R_{q} u_{x x}(x), \quad R_{q}=L \frac{s}{v_{F}} a \Phi(a), \quad a \equiv \frac{v_{F}}{s} \frac{\omega \tau}{\omega \tau+i}(10)
$$

Let us now evaluate the $K$ contribution. According to what we have said, it is given by the integral

$$
e \varphi_{K}(x)=\int_{C} \frac{d k}{2 \pi}\left(U_{x x}^{S}\right)_{k} R_{k} \exp (i k x)
$$

over a contour $C$ passing along the edges of the branch cut $k=$ $y \widetilde{\omega} / v_{F}(1 \leq y<\infty)$ in the complex $k$ plane. Using formulas $(6),(7)$, and (9), we can write this integral in the form

$$
\begin{aligned}
e \varphi_{K}(x) & =i \omega u(0) \frac{L}{v_{F}} \int_{1}^{\infty} d y \frac{y a^{2}}{a^{2}-y^{2}}\left[\left(y+\frac{1}{2} \ln \frac{y-1}{y+1}\right)^{2}+\frac{\pi^{2}}{4}\right]^{-1} \\
& \times \exp \left(\frac{i y \widetilde{\omega} x}{v_{F}}\right) \equiv i \omega u(0) \frac{L}{v_{F}} J(a, x) .
\end{aligned}
$$

We note that the $K$ contribution at any $x$ is proportional to the displacement of the surface, $u(0)$, while the $q$ contribution is proportional to the local deformation $u^{\prime}(x)$. This means that upon a variation of the surface potential by means of a capacitor with a vacuum (gas-filled) gap — an elastically free surface - we can record only the $K$ contribution. In the general case, by adding (10) and (12) together we obtain for the potential measured on the surface:

$\varphi(0)=\varphi_{q}(0)+\varphi_{K}(0)=\frac{i L}{e v_{F}}\left(\frac{2 I}{\rho s}\right)^{1 / 2}\left[J(a, 0)-\frac{a \Phi(a)}{C}\right](13)$

where $I$ is the energy flux in the sound beam, and $\rho$ is the density of the conducting medium.

Let us estimate the expected value of the effect. The coefficient in formula (13) at $L \sim 10 \mathrm{eV}$ and $I \sim 10 \mathrm{~W} / \mathrm{cm}^{2}$ has a value close to $\simeq 1 \mu \mathrm{V}$. The dependence of the functions $|a \Phi(a)|$ and $|J(a, 0)|$ on the parameter $\omega \tau$ is presented in Fig. 1 In the nonlocal region of frequencies and temperatures $(q l>1)$ the first of these rapidly approaches its limiting value $|a \Phi(a)|=\pi / 2$, whereas the second varies as $\ln |a|$; for $\omega \tau \gg 1$ its limiting value $\left|J\left(v_{F} / s, 0\right)\right| \approx \ln \left(v_{F} / s\right)$, which is practically always greater than $\pi / 2$ (the curves in Fig. 1. were constructed for the parameter value $v_{F} / s=200$ $\left(\ln v_{F} / s=5.3\right)$, which is typical of gallium). Thus the expected value of the effect is at the level of a few microvolts, i.e., quite amenable to measurement.

In Fig. 11 we see that for $C \sim 1$ the contributions under discussion become equal at $q l \sim 1$. The phases of these contributions, however, are shifted by $\pi / 2$, and therefore on the curves of the temperature dependence of $\varphi(T)$ one should expect an appreciable change in the phase of the signal in the temperature region where the mean free path becomes comparable to the wavelength of the sound.

Of course, the specular boundary condition used does not fully correspond to the experimental situation; however, it is known that taking more realistic boundary conditions (the 

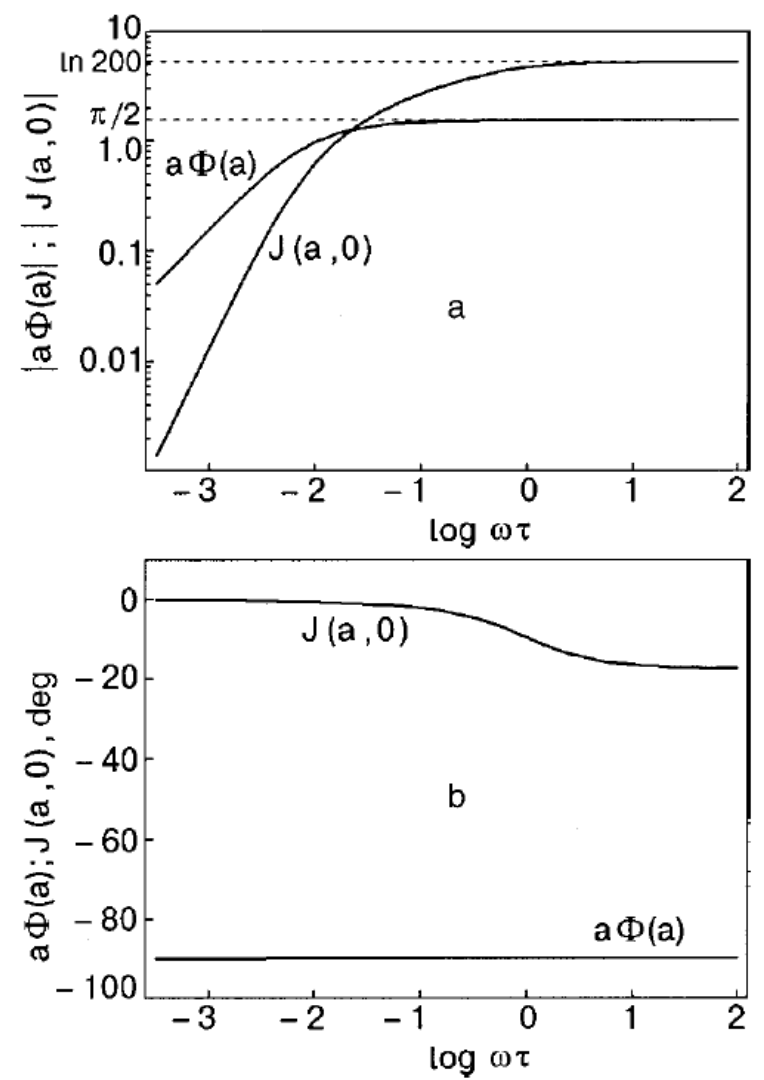

FIG. 1: Theoretical dependence of the amplitude (a) and phase (b) of the periodic $(\Phi)$ and aperiodic $(J)$ contributions to the surface electric potential, as functions of the scattering parameter.

diffuseness of the boundary) into account in nonlocal problems of acoustoelectronics complicates the calculations significantly without leading to any substantial changes in the results.$^{10}$ It can be hoped that in the given case the behavior of $\varphi(0)$ will correspond qualitatively to the behavior that follows from (13).

At the parameters values used, the amplitude of the adiabatic component of the charge density wave accompanying the propagation of a sound wave in a metal is of the order of $10^{8} \mathrm{~cm}^{-3}$. Let us now estimate the contribution of the nonequilibrium potential $\varphi(x)$ to the amplitude of the wave of uncompensated charge density. In the general case one can find its value by solving Eq. (5) jointly with Poisson's equation $k^{2} \varphi_{k}=4 \pi e\left\langle\psi_{k}\right\rangle$. As a result, for the nonequilibrium admixture to the charge density we obtain

$$
\left\langle\psi_{k}\right\rangle=-\left(u_{x x}^{S}\right)_{k}\left\langle\frac{\omega \Lambda_{x} x}{k v_{x}-\widetilde{\omega}}\right\rangle\left(1+\frac{4 \pi e^{2}}{k^{2}}\left\langle\frac{k v_{x}}{k v_{x}-\widetilde{\omega}}\right\rangle\right)^{-1}
$$

The final answer depends on the relationship between the squares of the inverse screening radius and the actual wave number. For the periodic component of the charge density wave $\left(q r_{D} \ll 1\right)$ the 1 in the denominator of (14) can be neglected at any distance from the surface of the metal, i.e., the answer reduces to a double differentiation of the potential $\varphi_{q}$ found previously from the condition of electrical neutrality. As a result, the amplitude of this component turns out to be small $\left(\sim s / v_{F}\right)$ in comparison with the adiabatic contribution.
To estimate the $K$ contribution at distances $x \gg r_{D}$ from the surface this approximation is again applicable, since in this case the convergence of the integrand in (12) (which acquires an additional factor of $k^{2}$ after the double differentiation with respect to $x$ ) at large $k$ is provided by the exponential factor, and the unity in the denominator of (14) can again be neglected. This allows us to obtain the following estimate for the uncompensated charge of the $K$ component at distances from the surface which are small compared to the mean free path and the wavelength of the sound:

$$
\delta n(x)-i \omega u(0) \frac{L}{v_{f}} \frac{q^{2}}{4 \pi e^{2}} \ln \frac{v_{F}}{x \widetilde{\omega}} .
$$

Thus the uncompensated charge of the $K$ component increases logarithmically as $x \rightarrow 0$, reaching a maximum value in the surface region $x \leq r_{D}$. The density of this surface charge can be estimated to logarithmic accuracy by employing a cutoff at $k \sim r_{D}^{-1}$ in the total denominator of expression (14) and making the substitution $x \rightarrow r_{D}$ in Eq. (15); this leads to a value $\delta n \sim 10^{8} \mathrm{~cm}^{-3}$, which is comparable to the amplitude of the adiabatic component.

We conclude this Section by verifying the applicability of the reciprocity theorem for the "exotic" mechanism of acoustoelectric coupling under consideration. In this case the acoustoelectric converter would be of the electrostatic type, in which the ponderomotive forces are due to the interaction of the electric charges. For converters of this type the electromechanical reciprocity theorem can be formulated as ${ }^{11}$

$$
\frac{\partial F}{\partial g}=\frac{\partial \varphi}{\partial u}
$$

where $F$ is the density of the mechanical force, which is causally related to the uncompensated charge density $g$, while the remaining components are the same as those already used. The relation between the electric field and the periodic elastic displacement is written in the form $\mathbf{u}=B \mathbf{E}$. Since $F=\rho s^{2} d^{2} u / d x^{2}$ and $g=\operatorname{div} \mathbf{E} / 4 \pi$ (since we are seeking an harmonic component of the displacements, differentiation with respect to $x$ reduces to multiplication by $q$ ), and, using formula (12) for a free surface $(C=\infty)$, we find $B \approx$ $L \ln \left(v_{F} / s\right) /\left(4 \pi \rho \operatorname{sev}_{F}\right)$, which agrees exactly with the result obtained in Ref. 6 .

\section{EXPERIMENTAL STUDY OF THE SOUND-GENERATED SURFACE POTENTIAL IN CONDUCTORS FOUND IN THE NORMAL STATE}

The basic scheme of the experiment is illustrated in Fig. 2. The sample under study is used to short a coaxial feeder, the outer conductor of which is in contact with the sample outside the region of incidence of the sound beam. The inner, springloaded conductor is a fragment of a hemisphere of rather large radius $(\sim 1 \mathrm{~cm})$. Thus the experiment measures the amplitude and phase of the potential difference between the central part of the "hot" spot created by a sound beam of diameter $\sim 4 \mathrm{~mm}$ and the remote parts of the surface of the sample at zero potential. In some experiments the galvanic contact of the inner 


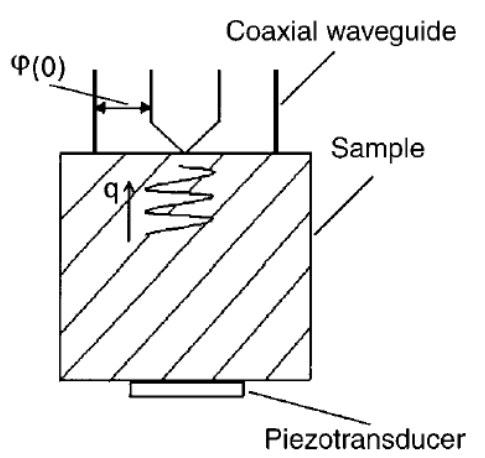

FIG. 2: Diagram of the experiment.

conductor of the coaxial feeder with the sample was replaced by a capacitive coupling $(C \sim 5 \mathrm{pF})$, or in some cases a flat (planar) coil displaced relative to the axis of symmetry of the sound beam was used to detect the magnetic field of the currents spreading out from the "hot" spot (this will be referred to below as an asymmetric coil).

An rf oscillator $(\omega / 2 \pi \sim 55 \mathrm{MHz})$ and a lithium niobate piezotransducer were used, giving a maximum acoustic power per pulse of up to $50-100 \mathrm{~W} / \mathrm{cm}^{2}$. The pulse duration $(\sim 5 \times$ $\left.10^{-7} \mathrm{~s}\right)$ and the pulse repetition rate $(\sim 17 \mathrm{~Hz})$ were chosen so that the heating of the sample at a temperature $T \sim 1 \mathrm{~K}$ did not exceed $(2-3) \cdot 10^{-2} \mathrm{~K}$.

Gallium. Single-crystal samples of high-purity gallium were used, ensuring a parameter value $\omega \tau \sim 5$ at the working frequency in the region of impurity scattering.

When the potential was registered using the galvanic contact, in addition to the signal coincident in time of arrival with the acoustic pulse, an electron-sound signal, passing through the sample at the Fermi velocity, was also observed (for temporal separation of the latter from the signal appearing at the time of the probe pulse, a germanium delay line, not shown in Fig. 2. was used). Analysis of the nature of the electron sound is proposed as the subject of a separate study; in this paper we discuss only the electric potential arising on the surface of the sample at the time of arrival of the sound pulse.

The maximum response at a fixed excitation power could be attained only for freshly ground surfaces; prolonged storage of a sample led to a falloff of the signal amplitude, apparently because of diffusion of impurities into the subsurface region. At $T=1.5 \mathrm{~K}$ the value of $|\varphi(0)|$ at the maximum excitation power reached $30-50 \mu \mathrm{V}$. The characteristic value of the Fermi velocity on the main sheets of the Fermi surface of gallium is $v_{F} \approx 7 \times 10^{7} \mathrm{~cm} / \mathrm{s}, 12$ and therefore in accordance with the estimate (13) a large value of the deformation potential $(L \approx 20 \mathrm{eV})$ is required in order to obtain such values of $\varphi(0)$. It is also possible that the main contribution to the effect is given by sheets of the Fermi surface on which the Fermi velocity is low, which are known to exist in gallium. 12

Typical curves of the temperature dependence of the amplitude and phase of the potential measured with the use of a galvanic contact are presented in Fig. 3. The data for $|\varphi(0)|$ are corrected for the change in sound attenuation in the sample. The resultant phase of the signals is determined not only by the phase of $\varphi(0)$ but also, and mainly, by the acoustic delay. However, in the investigated temperature interval the
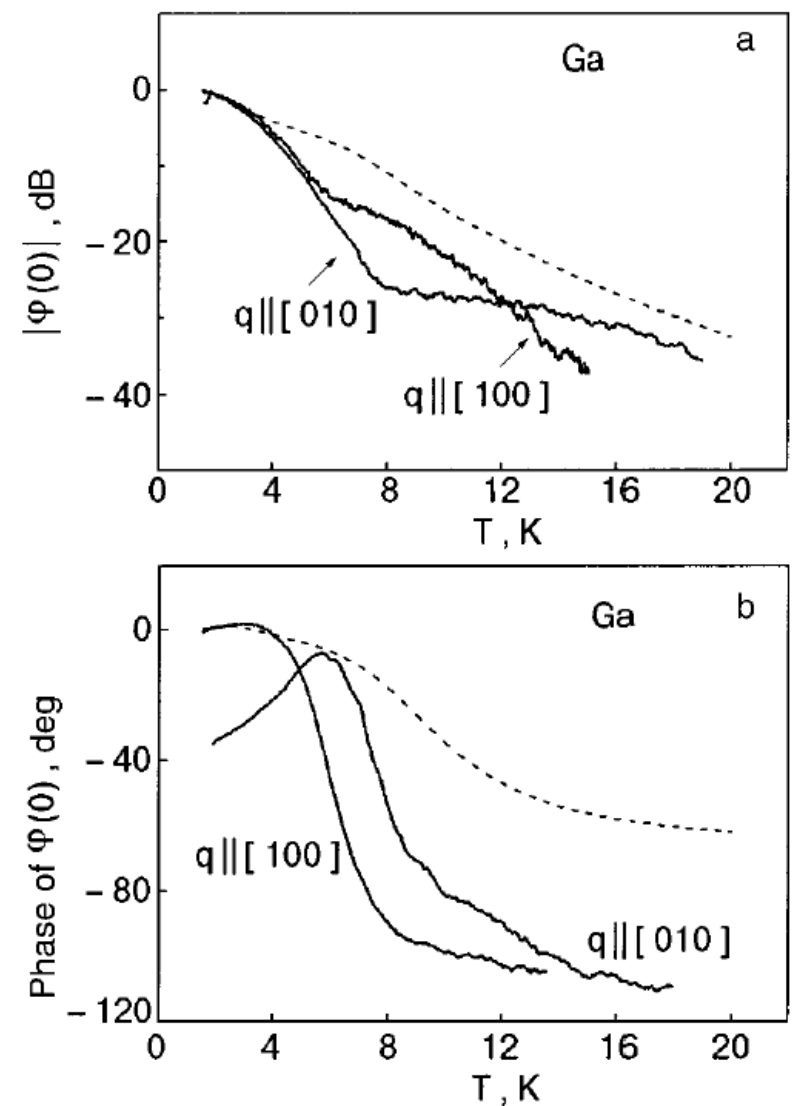

FIG. 3: Amplitude (a) and phase (b) of the potential $\varphi(0)$ measured with a galvanic contact on $\mathrm{Ga}$ in the propagation of sound along different crystallographic directions (solid curves) at a frequency of 55 $\mathrm{MHz}$. The dashed curve shows the result of a calculation according to Eq. (13) for $C=1,(\omega \tau)^{-1}=0.2+0.05 T^{2}+0.15 T^{3}, v_{F} / s=200$.

corrections due to the change in the sound velocity did not exceed a few percent of the measured total variations of the phase.

For $\mathrm{Ga}$ the temperature dependence of the relaxation time is known quite well, 13 and therefore in Fig. 3 we also show the calculated curves obtained at $C=1$. The calculations were done on the assumption that low-angle scattering is efficient $\left[(\omega \tau)^{-1}=0.2+0.05 T^{2}+0.15 T^{3}\right]$, although this question requires separate investigation. Without going into the details of the anisotropy of the effect, which is clearly seen on the experimental curves, we will mention the two circumstances that we think are essential.

1. As expected from the analysis given above, the phase of $\varphi(0)$ experiences significant $(\sim \pi / 2)$ variation in the temperature region corresponding to the transition from the nonlocal to the local regime. On the whole, the scale of the variations of the amplitude and phase of $\varphi(0)$ agree with the calculation. There is justification for thinking that the stated theoretical ideas about the nature of the origin of $\varphi(0)$ and the decisive role of the $K$ contribution at large values of $q l$ are in qualitative agreement with experiment. ${ }^{18}$

2. The calculation gives a much smoother variation of the amplitude and phase of $\varphi(0)$ in the crossover region than is observed in experiment. The kink in the temperature dependence of the amplitude, which coincides with the center of the "jump" in phase, cannot be described using Eq. (13), 
even when the model parameters are varied over wide limits. Apparently, the rate of decrease of the $K$ contribution in the crossover region is substantially higher than is predicted by Eq. (13). This circumstance is probably due to the "specularity" and isotropicity of the dispersion relation which were imposed on the model, and the solution for a more realistic case will be closer to the observed behavior of $\varphi(0)$.

Let us also mention some other features of the behavior of the phase of $\varphi(0)$. For $q \|[010]$ at $T<6 \mathrm{~K}$ the phase of the signal increases with increasing temperature (Fig. 3b). This behavior occurs only in that geometry, and it is most likely due to the broad flattening on the Fermi surface of $\mathrm{Ga}$ in the $\mathbf{q} \cdot \mathbf{v}=0$ region ${ }^{14}$ It is easy to see from the relations given above that in this case, for $\omega \tau \sim 1$, the increase in the scattering leads to growth of the phase of the $q$ contribution while having practically no effect on the $K$ contribution. The model calculation according to Eq. (13) with the actual relative area of the flat part taken into account $(\sim 2-4 \%$; Ref. 14$)$ gives a good description of both the scale of this effect and its temperature dependence.

It follows from Fig. 3b that the change in phase of the signal in the crossover region is assuredly in excess of $\pi / 2$. This is possible due to the complex nature of the parameter $C$ in the case of reflection of sound from a contact region of small size. If $C$ in Eq. (13) has the form $C=C_{0}(1+i \beta)(\beta>0)$, then in the nonlocal parameter region, where the contribution from the first term in (13) predominates, the influence of $\beta$ is insignificant. However, on going to the local limit the phase of the harmonic component turns out to be lower than the calculated value by an amount $\arctan (\beta)$.

In the proposed conception of the origin of $\varphi(0)$ the variation of the phase of the recorded signal is due to the fact that the interface in the contact region is elastically non-free $\left(u^{\prime}(0) \neq 0\right.$, i.e., $\left.C^{-1} \neq 0\right)$. To check this idea, we replaced the galvanic contact with a capacitor with a vacuum gap (more precisely, a gap filled with a heat-exchange gas). The result is shown in Fig. 4. In view of the small value of the capacitance (as we were striving to avoid edge effects, the electrode forming the capacitor had a diameter of $\sim 2 \mathrm{~mm}$ ) we were unable to make measurements in the same temperature interval as in Fig. 3. Nevertheless, it is reliably established that in this case there is no significant decrease of the phase of the signal. We assume that this result, taken together with the data of Fig. $3 \mathrm{~b}$, is unambiguous evidence in support of the approach developed here. Moreover, relation (12) also predicts a certain increase in the phase of the $K$ contribution with increasing scattering even in the purely isotropic approximation; this is apparently registered by the capacitive sensor, although on a larger scale (Fig. [4b).

The presence of excess surface charge in the region of the "hot" spot presupposes the existence of currents spreading out from the center of the sample toward the periphery. To detect them we used a flat coil asymmetrically shifted relative to the center of the sample. The plane of the turns of this coil were oriented perpendicular to the interface and parallel to the radial direction, the surface of the sample in the region of the "hot" spot was left elastically non-free. The results of this experiment are also presented in Fig. 4. The amplitude of the
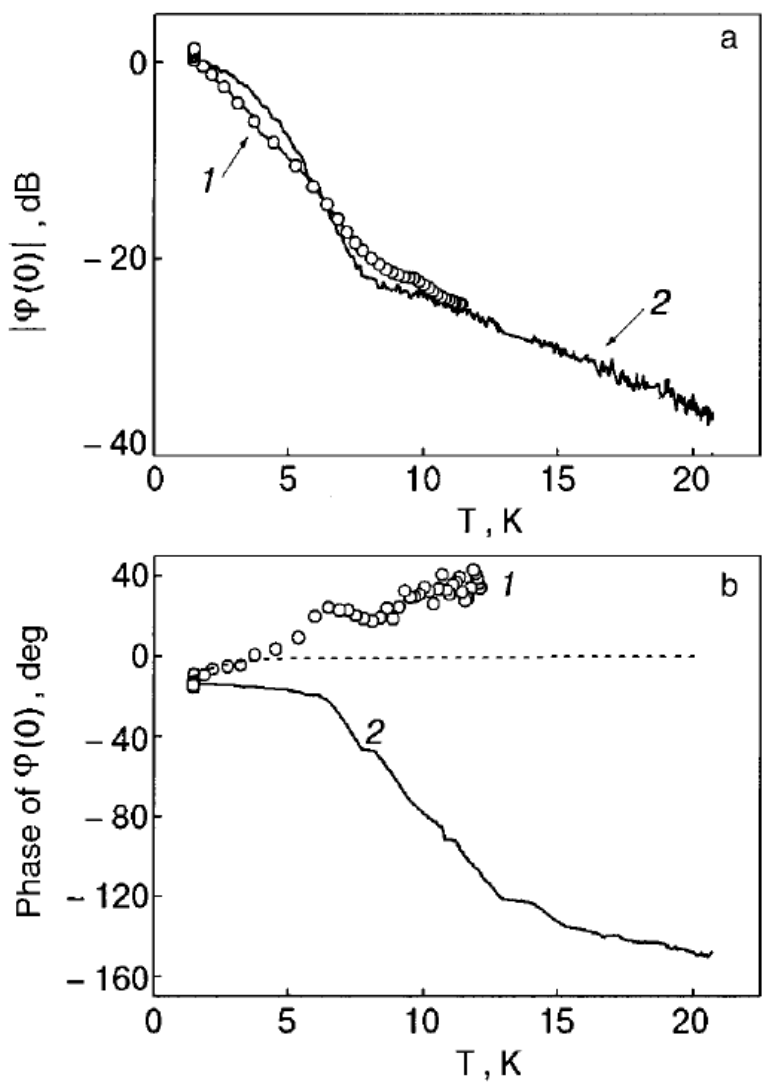

FIG. 4: Amplitude (a) and phase (b) of the potential $\varphi(0)$ measured on Ga with a capacitive sensor (1) and an asymmetric coil (2) (solid curves). The dashed curves show the results of a calculation according to Eq. (12) (the parameters used in the calculation were the same as in Fig. 3).

signal behaves analogously to that shown in Fig. 3 3 , while the phase of the signal deviates even more to the high side of $\pi / 2$. Most likely this is due to the fact that the rf conductivity of the metal, being a complex quantity, varies in phase on going to the local limit, thereby altering the phase of the rf current as well, so that the total shift increases.

Tungsten and aluminum. In tungsten in the impurityscattering region the value of the parameter $\omega \tau$ is approximately equal to 1 , while in aluminum $\omega \tau \approx 0.3$. It should be noted, however, that our estimates of the impurity scattering were obtained from a study of the bulk characteristics and can be somewhat overestimated for the surface regions. The values of $|\varphi(0)|$ measured in these metals turned out to be substantially smaller than in gallium $(\sim 1 \mu \mathrm{V})$. This is clearly due to the small value of the deformation potential, since a slight (see Fig. 1) increase in the scattering (i.e., decrease in the parameter $\omega \tau$ in comparison with gallium) should not lead to a substantial decrease in $|\varphi(0)|$.

Curves of the temperature dependence of the amplitude and phase of $\varphi(0)$ for $\mathrm{W}$ and $\mathrm{Al}$ are shown in Fig. 5. In spite of the small value of the detected signal, the curves for tungsten qualitatively repeat the behavior of $\varphi(0)$ in $\mathrm{Ga}$, the most salient feature of which, in our view, is the significant variation of the phase of the signal. In $\mathrm{Al}$ the phase of the signal is practically unchanging. It can be supposed that, as in $\mathrm{Ga}$, the more rapid (in comparison with Eq. (13)) falloff of the $K$ con- 

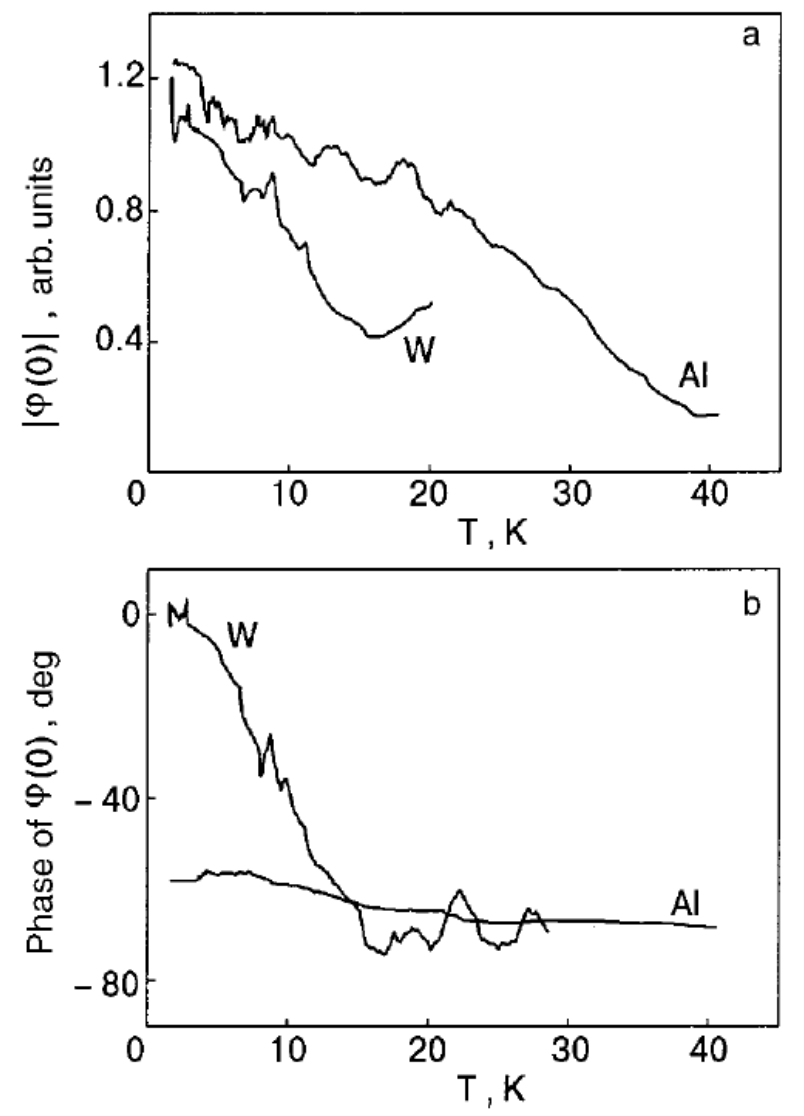

FIG. 5: Amplitude (a) and phase (b) of the potential $\varphi(0)$ measured with a galvanic contact on $\mathrm{W}$ and $\mathrm{Al}$.

tribution has led to its nearly complete suppression already at the starting (impurity) value of $\omega \tau$.

\section{INFLUENCE OF THE SUPERCONDUCTING TRANSITION ON $\varphi(0)$}

Before turning to a description of the experimental results, let us briefly present the theoretical scheme for estimating the possible behavior of $\varphi(0)$ below $T_{C}$. It follows from the above discussion that the existence of a measurable potential $\varphi(0)$ is due to the deformation interaction of the electrons with the elastic field of a longitudinal wave. In a superconductor only normal excitations interact with sound through the deformation potential. The "freezing out" of the normal excitations leads to the situation that for $T \ll T_{C}$ the potential $\varphi(0)$ is due solely to the Stewart-Tolman inertial field, which we are neglecting in this paper. Consequently, our problem consists in estimating the decay law of $\varphi(0)$ below $T_{C}$.

In a superconductor the electromagnetic field is customarily described by a gradient-invariant combination of $\Phi$ (the electrochemical potential of the excitations) and $\mathbf{p}_{s}$ (the momentum of the superconducting condensate) and the spatial and time derivatives of the phase of the order parameter $\chi$ and the electromagnetic potentials $\varphi$ and $\mathbf{A}$ :

$$
\Phi=\frac{1}{2} \frac{\partial \chi}{\partial t}+e \varphi, \quad \mathbf{p}_{s}=\frac{1}{2} \nabla \chi-\frac{e}{c} \mathbf{A} .
$$

In Ref. 15, which is devoted to the general theory of elasticity in superconductors, a relation is obtained between the fields $\Phi$ and $\mathbf{p}_{s}$ and the elastic displacements $\mathbf{u}$. In the case of longitudinal sound and an isotropic one-dimensional model, one can, using Ref. 15, write the following relations for the Fourier components of $\Phi$ and $p_{s}$ :

$$
\begin{aligned}
& -a \Phi=c p_{s x}+i \omega c^{(d)} m u_{x}, \\
& -\left(a b+c^{2}\right) p_{s x}=i \omega\left[a b^{(d)}-c c^{(d)}\right] m u_{x} .
\end{aligned}
$$

The solution of the boundary-value problem analogous to that considered above but for superconductors meets with considerable difficulties due to the energy dependence of the velocity of normal excitations, $\underline{16}$ and therefore for making estimates we limit consideration to the $q$ contribution only, for which the wave vector of the Fourier component coincides with the wave vector of the sound. Asymptotic expressions for the polarization coefficients $a, b$, and $c$ of the electron subsystem and the electroacoustic coefficients $c^{(d)}$ and $b^{(d)}$, which were all found in Ref. 15 in the limit of strong spatial dispersion $(q l \gg 1)$, have the form

$$
\begin{aligned}
a & =1+\frac{i \pi}{2} \frac{s}{v_{F}} f(\Delta), \quad b=\frac{L}{m}\left[\rho_{s}-\frac{3 \pi i}{2} \frac{s}{v_{F}} \frac{\Delta / 4 T}{\cosh ^{2}(\Delta / 4 T)}\right], \\
c & =s\left(1-\rho_{s}\right), \quad b^{(d)}=\left[\frac{i \pi}{2} \frac{s}{v_{F}} \frac{L}{m}\left(1+\frac{\Delta}{2 T} \ln \frac{T v_{F}}{\Delta s}\right),\right. \\
c^{(d)} & =-\frac{i \pi}{2} \frac{L}{m v_{F}} f(\Delta),
\end{aligned}
$$

where $\rho_{s} \approx 2\left(T_{C}-T\right) / T_{C}$ is the density of the superconducting condensate, $f(\Delta)=2 /(\exp (\Delta / T)+1)$ is the Fermi function, and $\Delta(T)$ is the superconducting gap.

Restricting consideration in (18) and (19) to the first nonvanishing terms of the expansion in the small parameter $s / v_{F}$ and assuming that the inequalities $\rho_{s}, \Delta / T>\left(s / v_{F}\right)^{2}$ hold, we find

$$
\begin{aligned}
& \Phi=i \omega \frac{c b^{(d)}-b c^{(d)}}{a b+c^{2}} m u_{x}=-i \omega c^{(d)} m u_{x}, \\
& p_{s}=-i \omega \frac{a b^{(d)}+c c^{(d)}}{a b+c^{2}} m u_{x}=-i \omega \frac{b^{(d)}+s C^{(d)}}{b} m u_{x} .
\end{aligned}
$$

In the case of longitudinal sound the vector potential in (17) can be dropped, and we obtain for the electric potential

$$
e \varphi(x)=\Phi(x)-s p_{s}(x) .
$$

It follows from (19)-(21) that the contribution $p_{s}$ to the periodic component of the potential is small in the parameter $s / v_{F}$ and can also be dropped, and the relation between the amplitudes of the potential and the normal $(n)$ and superconducting $(s c)$ states has the form

$$
(e \varphi)_{s c}=(e \varphi)_{n} f(\Delta),
$$

i.e., the $q$ contribution should fall off with decreasing temperature in the same way as the sound attenuation coefficient. In addition, it follows from (22) that the phase of the potential should not change on transition through $T_{C}$. Since the $K$ 

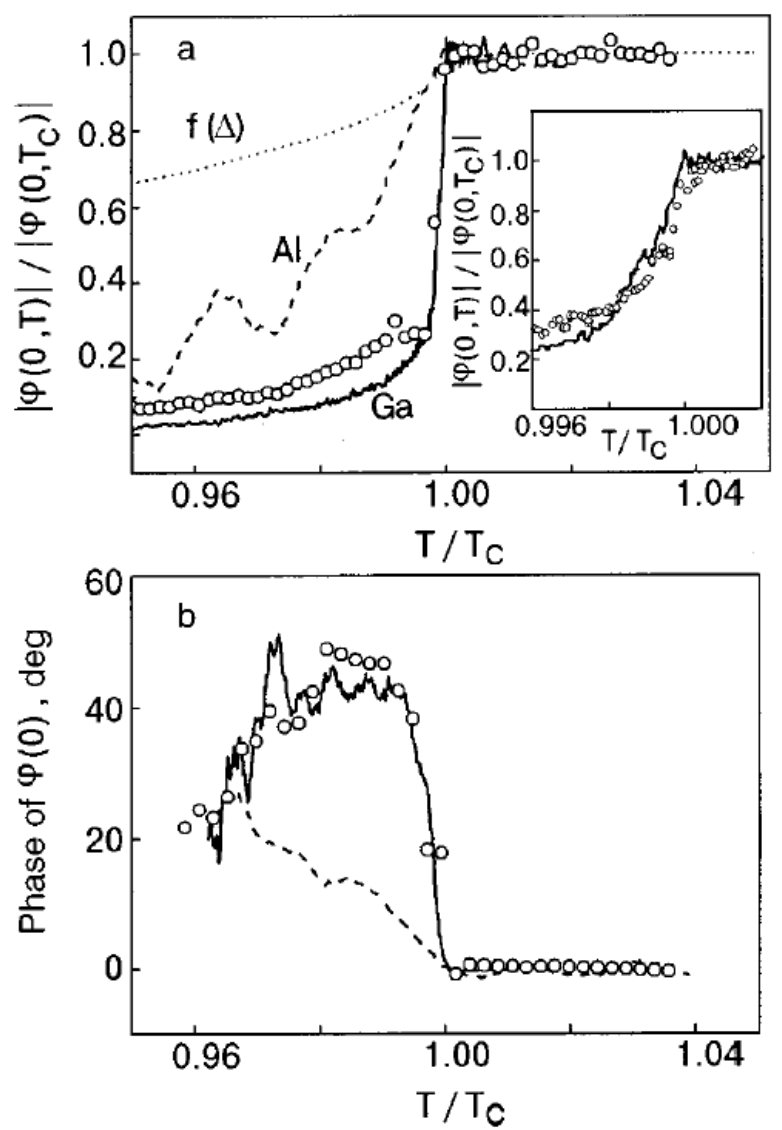

FIG. 6: Effect of the superconducting transition on the electric potential: amplitude $|\varphi(0)|$ - the solid and dashed curves are for a galvanic contact ( $\mathrm{Ga}$ and $\mathrm{Al}$ ), the circlets are for an asymmetric coil (Ga), and the upper dotted curve shows $f(\Delta)$. Inset: the behavior of $|\varphi(0)|$ in $\mathrm{Ga}$ (galvanic contact) for different excitation amplitudes: solid curve $-+10 \mathrm{~dB}$, circlets $-0 \mathrm{~dB}$ (a); the variation of the phase $\varphi(0)$ below $T_{C}$ : the solid and dashed curves are for a galvanic contact ( $\mathrm{Ga}$ and $\mathrm{Al}$ ), and the circlets are for an asymmetric coil (b).

component of the potential is also due to the deformation interaction, there is no reason to think that its law of variation will be substantially different from (22).

The experimental results presented in Fig. 6 strongly contradict the estimates given above. Even in Al, in which apparently only the periodic component of the signal is present to a significant degree, the amplitude of $\varphi(0)$ changes much more sharply than $f(\Delta)$, and the phase undergoes a rather rapid rise. These features are more pronounced in $\mathrm{Ga}$, in which one might suspect a manifestation of some sort of nonlinearity, since a nonlinearity is expressed quite clearly in the sound attenuation. $\stackrel{17}{=}$ For an excitation intensity close to the maximum, nonlinear behavior of $\varphi(0)$ was indeed observed, associated with both overheating and a sharp drop in the sound attenuation coefficient below $T_{C}$, with a corresponding decrease in the heat release. The data presented in Fig. 6a pertain to the region of amplitudes in which these effects are practically absent (inset in Fig. 6a).

The following "simplified" line of reasoning is also possible. In the experiments described, the potential difference between the "hot" spot and the remote parts of the sample is recorded in the regime of spreading surface currents. There- fore, the value of the potential that is registered can depend on the spreading resistance. In other words, in a superconductor the rapid growth of the diamagnetic contribution to the conductivity $\left(\sim \rho_{s} v_{F} / s\right)$ can lead to "short-circuiting" of the source of the electromotive force, and that is what is detected in the experiment. In Fig. 6 we also show the signal taken from the asymmetric coil, which registered the amplitude and phase of the spreading currents specifically. We see that the amplitude of this signal falls off just as rapidly as does $|\varphi(0)|$, and the phase also increases practically in a jump. Therefore, from the standpoint of the "simplified" explanation a rapid growth of the "resistance" of the source of emf must also be assumed, and that is hard to imagine.

From the results presented in Fig. 6a for Ga we see that beyond the region of very rapid drop of $|\varphi(0)|$ comes a more gradual "tail," reminiscent of the evolution of $f(\Delta)$. This suggests another hypothesis: for some reason that we have not discussed, the $K$ contribution in a superconductor actually vanishes very rapidly, and the remaining $q$ contribution falls off as follows from the theory. We recall, however, that the phase of the $q$ contribution is different from the phase of the $K$ contribution by more than $\pi / 2$ (Fig. 3b), and so this scenario should be accompanied by a downward phase jump of corresponding amplitude. The data of Fig. 6b are inconsistent with this hypothesis as well.

At present the authors do not have any reasonable explanation for the described behavior of $\varphi(0)$ near $T_{C}$. On the whole, the picture looks as if either a state in which an uncompensated charge cannot exist develops with catastrophic rapidity in a superconductor which is found in the normal phase, at least in the nonlocal limit, or else below $T_{C}$ the electric potential just as rapidly transforms to a quantity that cannot be measured by a voltmeter. We add that a jump in the phase of $\varphi(0)$ by approximately $\pi / 4$ can be evidence that the surface impedance has some relation to the observed effect, since the penetration depth of the field at the transition through $T_{C}$ changes from a complex quantity with approximately equal real and imaginary parts (their relative sizes depend on whether the normal or anomalous skin-effect regime is realized) to the purely real London penetration depth.

\section{CONCLUSION}

In summary, we have carried out a theoretical analysis and experimental observation and study of the electric potential arising when a longitudinally polarized elastic wave is incident normally on a metal surface. The potential under study is the sum of two contributions. The first of them, the $q$ contribution, is due to forced oscillations of the electric field, which is proportional to the elastic deformation $u^{\prime}$, i.e., it can be detected only on a non-free boundary, where $u^{\prime}(0) \neq 0$. The second, $K$ contribution is due to the presence of the metal boundary itself, which distorts the ballistic motion of nonequilibrium carriers in a subsurface layer of thickness $\sim l$; its amplitude turns out to be proportional to the displacement of the surface, $u(0)$. In the nonlocal limit the amplitude of the $K$ contribution is markedly greater than that of the $q$ contribu- 
tion, while in the local limit it is the other way around. The phase of the $K$ contribution leads the phase of the $q$ contribution by approximately $\pi / 2$, and therefore in the crossover region the phase of the recorded potential varies quite sharply. The experimental observation of just such behavior is, in the opinion of the authors, an unambiguous qualitative confirmation of the correctness of the ideas developed in this paper about the origin of the potential in question.

At the superconducting transition the amplitude of the potential falls in a catastrophically rapid manner, and the phase just as rapidly increases by approximately $\pi / 4$. This behavior is inconsistent with the theoretical concepts, which predict a much smoother decrease of the amplitude, close to the BCS dependence of the longitudinal sound attenuation coefficient, and the absence of any phase variations.

The authors express their deep gratitude to E. A. Masalitin for invaluable contribution to the development and preparation of the measuring apparatus, which was ideally suited for addressing the problems investigated in this study.
1 A. N. Vasil'ev and Yu. P. Gaĭdukov, Usp. Fiz. Nauk 141, 431 (1983) [Sov. Phys. Usp. 26, 952 (1983)].

2 This is not strictly accurate. Applications of electroacoustic conversion often make use of the circumstance that in a quasistatic electric field the surface of a conductor is subjected to an additional pressure. In an alternating field this pressure excites elastic displacements of the surface, including longitudinal ones. This effect is widely used in low-frequency acoustic experiments with vibrating reeds,,$\frac{3}{3}$ and it is also the basis of the ESAT (electrostatic acoustic transformation) mechanism. ${ }^{4}$. Since the response is quadratic in the field amplitude, in this case the reciprocity principle is, of course, not obeyed, and this effect can be used for acoustoelectric conversion only with additional "linearization". In the present paper we discuss only linear effects.

3 P. Esquinazi, J. Low Temp. Phys. 85, 3/4, 139 (1991).

${ }^{4}$ H. Shimizu and A. J. Bahr, IEEE Ultrasonic Symp. Proc. 17 (1976).

5 V. M. Gokhfel'd and V. D. Fil', Fiz. Tekh. Vysokikh Davlenii 11, 4, 76 (2001).

6 A. N. Vasil'ev, V. M. Gokhfel'd, and M. I. Kaganov, Zh. Éksp. Teor. Fiz. 92, 2283 (1987) [Sov. Phys. JETP 65, 1286 (1987)].

7 V. M. Kontorovich, Zh. Éksp. Teor. Fiz. 45, 1638 (1963) [Sov. Phys. JETP 18, 1125 (1964)].

8 V. M. Gokhfel'd, M. A. Gulyanskiŭ, and M. I. Kaganov, A. G. Plyavenek, Zh. Éksp. Teor. Fiz. 89, 985 (1985) [Sov. Phys. JETP
62, 566 (1985)].

9 A. I. Kopeliovich and M. S. Churyukin, Fiz. Nizk. Temp. 19, 176 (1993) [Low Temp. Phys. 19, 125 (1993)].

10 V. M. Gokhfel'd and M. I. Kaganov, Fiz. Nizk. Temp. 11, 517 (1985) [Sov. J. Low Temp. Phys. 11, 282 (1985)].

11 V. V. Furduev, Electroacoustics [in Russian], OGIZ, Moscow (1948).

12 E. V. Bezugly̌̆, N. G. Burma, E. Yu. Deĭneka, and V. D. Fil', Fiz. Nizk. Temp. 19, 667 (1993) [Low Temp. Phys. 19, 477 (1993)].

13 E. V. Bezuglyi, N. G. Burma, E. Yu. Deyneka, A. I. Kopeliovich, and V. D. Fil, J. Low Temp. Phys. 91, 179 (1993).

14 E. V. Bezugly̌̆, A. M. Stepanenko, and V. D. Fil', Fiz. Nizk. Temp. 13, 246 (1987) [Sov. J. Low Temp. Phys. 13, 140 (1987)].

15 E. V. Bezuglyi, Fiz. Nizk. Temp. 9, 15 (1983) [Sov. J. Low Temp. Phys. 9, 6 (1983)].

16 E. V. Bezuglyi and A. V. Boichuk, Fiz. Nizk. Temp. 23, 676 (1997) [Low Temp. Phys. 23, 507 (1997)].

17 V. D. Fil', V. I. Denisenko, and P. A. Bezugly̌̆, JETP Lett. 21, 329 (1975).

18 We note that for the customary way of representing the elastic field $[\sim \exp (-i \omega t+i \mathbf{q} \cdot \mathbf{r})]$, in comparing the measured phase variations with those calculated using relation (13), the sign of the latter should be changed. 